J. Bangladesh Acad. Sci., Vol. 42, No. 2, 137-153, 2018

DOI: https://doi.org/10.3329/jbas.v42i2.40041

\title{
IDENTIFICATION AND ANTIBIOGRAM STUDIES OF PATHOGENIC BACTERIA ISOLATED FROM DRINKING WATER (UNPACKAGED) IN CHITTAGONG CITY, BANGLADESH
}

\author{
AFRA NAWER MAESHA, LOLO WAL MARZAN* AND YASMIN AKTER \\ Department of Genetic Engineering and Biotechnology, Faculty of Biological Sciences, University of \\ Chittagong, Chittagong- 4331, Bangladesh
}

\begin{abstract}
Drinking water (unpackaged) samples were collected from twenty roadside shops of different locations in Chittagong metropolitan area, where physicochemical parameters ( $\mathrm{pH}$, TDS, temperature) were not exceeded WHO prescribed range in most cases. TVC, TCC, TFCC, TSC, Salmonella spp. and Vibrio spp. were found contaminated as $85 \%, 70 \%, 50 \%, 20 \%, 30 \%$ and $75 \%$, respectively. All the bacterial isolates $(\mathrm{n}=43)$ were found positive for $16 \mathrm{~S}$ rDNA gene, while 12 isolates were coliform positive identified by lacZ gene amplification. Nine bacterial genera were finally identified depending on biochemical characteristics and two of them were further confirmed by $16 \mathrm{~S}$ rDNA sequencing. Escherichia coli, Citrobacter spp., Klebsiella pneumoniae and Vibrio cholerae were resistant to amoxicillin, where all the bacterial isolates exhibited sensitivity to ciprofloxacin, gentamicin, imipenam and levofloxacin. Presence of human pathogenic and antibiotic resistant bacteria in unpackaged drinking water, suggested for increasing regular monitoring and public awareness to ensure public health safety.
\end{abstract}

Keywords: Microbial contamination, Klebsiella pneumoniae, Acinetobacter baumannii, Vibrio cholerae, antibiotic resistance.

\section{INTRODUCTION}

Water with microbial, chemical and physical characteristics that meet WHO guidelines is called safe drinking water and public health impacts depends on the quality of drinking water worldwide (Olaoye et al. 2009). Waterborne diseases are one of the major health problem in developing countries (Oyedeji et al. 2010) due to unsafe drinking water and unhygienic sanitary condition. Imbalance between demand and supply of drinking water had led to chronic shortages that resulted in pollution and environmental deterioration in Asian and African countries, where children (under five) are the most common victims of microbial diseases transmitted through water (Prasai et al. 2007). Microbial contamination in water causes majority of water related health problems according to WHO (2015). In Bangladesh, $80 \%$ of all diseases are caused by consumption of contaminated water which is responsible for one third of deaths (UNCED 1992 ) and $28 \%$ of children's death is attributed to waterborne diseases caused by pathogenic bacteria (Aziz et al. 1990).

Chittagong, is one of the densely populated urban areas of Bangladesh, has been suffering from various water processing problems along with insufficient drinking water supply (Zuthi et al. 2009), where the main source of drinking water is CWASA (Chittagong water supply and sewage authority) (Nawas et al. 2012). Failure of the disinfection processes of raw water treatment plant or improper

*Corresponding author: <marzan.geb@cu.ac.bd>. 
filtration of sewage also the causes of bacteriological contamination in drinking water supplies studied previously (Prasai et al. 2007). Along with pathogenic bacteria, indicator bacteria of faecal contamination must not be present in quality drinking water; hence its detection in drinking water also has highest importance and become an emerging problem throughout the world (Parvez et al. 2016). Presence of coliform bacteria in water indicate, possible presence of enteric pathogens and potential human fecal contamination, lack of disinfection process as well as lack of system integrity (Bej et al. 1990). In this city, a large number of people eat their meals in different roadside shops, restaurants and from food vendors, where they cannot mostly afford to have bottled safe drinking water; thus they depend on cheaper unpackaged drinking water available here. So, it is urgently needed to evaluate microbiological quality and antibiogram study of that drinking water to concern the authority to ensure availability of safe drinking water for local consumers. The objectives of this study include- isolation and enumeration of bacteria from drinking water samples, their characterization and identification up to genus level by established biochemical and molecular methods and finally determination antibiotic sensitivity in order to create public awareness to ensure human health safety.

\section{MATERIALS AND METHODS}

\section{Sampling area and collection of water samples}

Twenty different roadside shops in Chittagong metropolitan area were selected for this study between January to September, 2017 (Table 2). Samples $(n=20)$ were collected separately in two sets of pre-sterilized $50 \mathrm{~mL}$ falcon tubes; systematically labeled and cold chain maintained during shipment into the laboratory. Physicochemical and microbiological analysis was tested immediately after transportation and other samples were preserved in refrigerator (at $4{ }^{\circ} \mathrm{C}$ ) before commencement of analysis and during experiments. Hygienic and aseptic practices were ensured during sampling and further studies.

\section{Physicochemical analysis}

The digital pH meter (Hanna, Italy) was first standardized with buffer solution to determine their pH value (Oluwole et al. 2016) along with temperature. Total dissolved solid (TDS) of each sample was measured by using a portable digital TDS measuring meter (HM digital), after due calibration. All experiments were conducted at ambient temperature and carried out in three replications.

Screening and isolation of bacteria from water samples

Microbial population was enumerated using total viable count (TVC) method on nutrient agar (NA) plate by means of serial dilution $\left(10^{-1}\right.$ to $10^{-7}$ ) following Addisu and Kiros (2016). After incubation $\left(37^{\circ} \mathrm{C}\right.$ for 24 hours $)$ the inoculated plates were screened for the discrete colonies of bacteria and population density (cfu/mL) was estimated (Rashed et al. 2013). Following enumeration, morphologically distinct colonies were screened by observing colony characteristics (gram nature, color, shape) using a binocular biological microscope (XSZ-107BN); then colonies of similar morphological features were grouped together (Beiranvand et al. 2017). Spread and pour plate 
techniques were adopted to isolate the colonies (Uddin et al. 2011), where pure culture was obtained by streak plate method on nutrient agar (pH 7.0) and EMB (Eosin-methylene blue) agar media. Thus selected, purified, cultured isolates were stored in the laboratory (at $-80^{\circ} \mathrm{C}$ ) in glycerol stock $(50 \%)$ solution for further studies.

Detection of total coliform, faecal coliform, Staphylococci spp. (TCC, TFCC, TSC)

Total coliform and faecal coliform count was detected by most probable number (MPN) technique (Cappuccino and Sherman, 2004) which calculated according to the statistical table described by Collee et al. (1996). Total Staphylococci count (TSC) was determined by spreading the purified colonies in manitol salt agar selective media, where yellow colonies were identificed as Staphylococcus aureus and estimated as Rashed et al. (2013).

Detection of Vibrio, Salmonella and Shigella spp

For the detection of Vibrio spp., thiosulphate citrate bile salt (TCBS) was used after preenrichment in alkaline peptone water and for Salmonella spp. Shigella spp., selective media salmonella shigella agar (SSA) was used (APHA 2001).

\section{Phenotypic and biochemical characterization of bacterial isolates}

For the identification of selected bacterial isolates, they were characterized by gram staining based on cultural, morphological; as well as biochemical characteristics as described in the Cowan and Steel's Manual (1974). For the activities of oxidase, catalase, citrate utilization, indole production, methyl-red (MR), voges- proskauer (VP) and triple sugar iron (TSI) tests isolates were biochemically analyzed (Williams 1989, Marzan et al. 2017) and provisionally identified according to Bergey's Manual of Systemic Bacteriology (Bergey et al. 1974).

Molecular identification of bacteria and total coliform

Genomic DNA was extracted from drinking water samples (Fatemeh et al. 2014, Sambrook et al. 1989) and stored them at $-20^{\circ} \mathrm{C}$. DNA concentration was measured by Thermo scientific Nano Drop 2000 spectrophotometer (Thermo scientific USA) following standard protocol. PCR and coliform detection of bacteria were carried out using previously published primers and targeted gene (Fatemeh et al. 2014; Mina et al. 2018). Primer specificity was determined by searching for similar sequences in microbial genome using the Basic Local Alignment Search Tool (BLAST). In each experiment, positive and negative control was carried out. Only samples, confirmed as coliform by MPN test were subjected to further confirmaction by lacZ amplification. A total composition of target gene, primer sequences, cycling parameters, PCR master mixture, amplicon size (bp) were performed for PCR amplifications in a thermal cycler (Nyx Technik) (Table 1).

\section{Analysis of amplified products}

Amplified PCR products were then analyzed by electrophoresis (Micro-Bio-Tech Brand) in 1\% (w/v) agarose gel in 1xTAE buffer, stained with ethidium bromide (1\%) (Sambrook et al. 1989), 
Table 1. Target genes, primer sequences, cyclic condition, PCR master mixture composition and amplicon size

\begin{tabular}{|c|c|c|c|c|c|}
\hline $\begin{array}{c}\text { Target } \\
\text { gene }\end{array}$ & Primer sequence (5 $\square \mathbf{- 3} \square$ ) & Cycling parameters & $\begin{array}{l}\text { Total Composition of } \\
\text { PCR master mixture }\end{array}$ & $\begin{array}{c}\text { Amplicon } \\
\text { size (bp) }\end{array}$ & Reference (s) \\
\hline $\begin{array}{l}\text { Common } \\
\text { bacterial: } \\
16 \mathrm{~S} \text { rDNA }\end{array}$ & $\begin{array}{l}\text { 8F-AGAGTTTGATCCTGGCTCAG } \\
\text { 850R-GACTACCAGGGTATCTAAT }\end{array}$ & $\begin{array}{l}5 \mathrm{~min} \text { at } 95^{\circ} \mathrm{C}, 35 \text { cycles of } \\
95^{\circ} \mathrm{C} \text { for } 40 \mathrm{~s}, 57^{\circ} \mathrm{C} \text { for } 50 \mathrm{~s} \\
\text { and } 72^{\circ} \mathrm{C} \text { for } 1 \mathrm{~min}\end{array}$ & $\begin{array}{l}\text { For } 10 \mu \mathrm{l}: 5 \mu \mathrm{l} \text { master } \\
\text { mix, } 2 \mu \mathrm{l} \text { template, } 1 \mu \mathrm{l}^{\mathrm{a}} \\
\text { and } 1 \mu \mathrm{l}^{\mathrm{b}}, 1 \mu \mathrm{l} \text { water }\end{array}$ & 800 & $\begin{array}{c}\text { Fouad } \text { et al. } \\
\text { 2002; } \\
\text { Mina et al. } 2018\end{array}$ \\
\hline $\begin{array}{l}\text { Coliform: } \\
\text { Lac Z }\end{array}$ & $\begin{array}{l}\text { F-ATGAAAGCTGGCTACAGGAAGGCC } \\
\text { R-CACCATGCCGTGGGTTTCAATATT }\end{array}$ & $\begin{array}{c}5 \mathrm{~min} \text { at } 95^{\circ} \mathrm{C}, 25 \text { cycles of } \\
95^{\circ} \mathrm{C} \text { for } 1 \mathrm{~min}, 60^{\circ} \mathrm{C} \text { for } 1 \\
\text { min and } 72^{\circ} \mathrm{C} \text { for } 1 \mathrm{~min}\end{array}$ & $\begin{array}{l}\text { For } 10 \mu \mathrm{l}: 5 \mu \mathrm{l} \text { master } \\
\text { mix, } 2 \mu \mathrm{l} \text { template, } 1 \mu \mathrm{l}^{\mathrm{a}} \\
\text { and } 1 \mu \mathrm{l}^{\mathrm{b}}, 1 \mu \mathrm{l} \text { water }\end{array}$ & 876 & $\begin{array}{l}\text { Fatemeh } \text { et al. } \\
\text { 2014; } \\
\text { Aghababaee } \text { et } \\
\text { al. } 2012\end{array}$ \\
\hline
\end{tabular}

$a=$ forward primer $; b=$ reverse primer $;=$ seconds

and compared with marker DNA (Gene Ruler 1 $\mathrm{kb}$ DNA Ladder) visualized under ultraviolet (UV) trans-illuminator (Benda company) and then photographed.

\section{Sequence analysis of $16 S$ rDNA PCR product}

Among nine, two $\left(\mathrm{L}_{2} \mathrm{C}, \mathrm{K}_{2} \mathrm{~S}\right)$ of biochemically identified (Bergey et al. 1974) bacterial isolates were then sent for sequencing (Biogen, South Korea) after purification. The results were then analyzed in the NCBI BLASTn program (https://blast.ncbi.nlm.nih.gov) and submitted to GenBank by using Bankit submission tool. Phylogenetic tree was constructed from the isolated $16 \mathrm{~S}$ rDNA bacterial sequence of $\mathrm{L}_{2} \mathrm{C}$ sample, with their mostly identical respective reference sequences from GenBank according to Saitou and Nei (1987).

\section{Determination of antibiotic sensitivity pattern}

Susceptibility of nine identified bacteria to different antimicrobial agents were then measured in vitro by employing the modified Bauer Kirby (1966) method. Commercially prescribed five antimicrobial discs [Amoxicillin (AML), Ciprofloxacin (CIP), Gentamicin (CN), Imipenem (IMP) and Levofloxacin (LEV)] (Himedia, India) were used for the tests.

\section{Statistical analysis}

Triplicate experiments were done in all the cases and the results expressed as mean value \pm standard deviation (SD). Data were captured into Microsoft Excel Software, version 2016 to calculate means and standard deviations.

\section{RESULTS AND DISCUSSION}

\section{Physicochemical analysis}

The $\mathrm{pH}$ values of drinking water samples $(\mathrm{n}=20)$ varied from $7.3 \pm 0.02-9.3 \pm 0.02$ (Table 2), where some of them $(n=6)$ were not within the WHO maximum permissible limits (6.5-8.5) for drinking water (WHO 2015). Most of the bacteria grow at neutral $\mathrm{pH}$ (Tasnim et al. 2010) and $\mathrm{pH}$ in water samples is reported to increase upon storage in Jordan (Chalchisa et al. 2017). TDS (total dissolved solids) values ranged from $64 \pm 0.01-251 \pm 0.10 \mathrm{ppm}$ for all samples which are also between recommended range of $<1000$ ppm (WHO 1996) (Table 2). Temperature affects the rate of chemical reactions in the water body which plays an important role in the survival of microorganisms (Akuffu et al. 2013). Recorded temperature in water samples were $15.8^{\circ} \mathrm{C}-23.6{ }^{\circ} \mathrm{C}$; where in most cases it does not exceed the WHO permissible limits of drinking water guidelines (Table 2). In previously it was reported that high 
temperature favored the re-growth of bacteria and after storage it can increase the number of faecal coliforms (Muyima and Ngcakani 1998). Sometimes, materials storage tank may affect the water temperature and water tank in shaded location can reduce temperature as well as growth of microorganisms and contamination (Chalchisa et al. 2017, WHO 2008).

Table 2. Sampling sites and physicochemical analysis of drinking water samples

\begin{tabular}{|c|c|c|c|c|c|}
\hline Sample no. & Sampling sites & Sample ID & pH value & TDS (ppm) & $\begin{array}{c}\text { Temperature } \\
\left({ }^{\circ} \mathrm{C}\right)\end{array}$ \\
\hline 1 & Anderkilla & $\mathrm{A}_{1}$ & $7.6 \pm 0.02$ & $198 \pm 0.20$ & 23.6 \\
\hline 2 & Agrabad & $\mathrm{A}_{2}$ & $7.3 \pm 0.02$ & $119 \pm 0.05$ & 17.0 \\
\hline 3 & Bakolia & $\mathrm{B}_{1}$ & $7.8 \pm 0.01$ & $152 \pm 0.20$ & 21.2 \\
\hline 4 & Chawkbazar & $\mathrm{C}_{1}$ & $7.8 \pm 0.01$ & $70 \pm 0.10$ & 15.8 \\
\hline 5 & Chandanpura & $\mathrm{C}_{2}$ & $* 9.3 \pm 0.02$ & $163 \pm 0.05$ & 20.5 \\
\hline 6 & 2 no. gate & $\mathrm{D}_{1}$ & $* 9.0 \pm 0.02$ & $107 \pm 0.05$ & 16.5 \\
\hline 7 & Ek-kilometer & $\mathrm{E}_{1}$ & $* 8.5 \pm 0.01$ & $166 \pm 0.20$ & 20.6 \\
\hline 8 & GEC circle & $\mathrm{G}_{1}$ & $7.4 \pm 0.01$ & $251 \pm 0.10$ & 18.5 \\
\hline 9 & Hathazari & $\mathrm{H}_{1}$ & $* 8.9 \pm 0.02$ & $172 \pm 0.2$ & 20.6 \\
\hline 10 & Kapashgola & $\mathrm{K}_{1}$ & $* 8.5 \pm 0.01$ & $137 \pm 0.02$ & 23.4 \\
\hline 11 & Kalurghat & $\mathrm{K}_{2}$ & $8.4 \pm 0.20$ & $64 \pm 0.01$ & 18.9 \\
\hline 12 & Khulshi & $\mathrm{K}_{3}$ & $8.2 \pm 0.03$ & $108 \pm 0.10$ & 21.5 \\
\hline 13 & Laldighi & $\mathrm{L}_{1}$ & $* 8.9 \pm 0.01$ & $166 \pm 0.05$ & 18.3 \\
\hline 14 & Lalkhan Bazar & $\mathrm{L}_{2}$ & $7.3 \pm 0.02$ & $184 \pm 0.01$ & 21.3 \\
\hline 15 & Muradpur & $\mathrm{M}_{1}$ & $* 8.5 \pm 0.01$ & $81 \pm 0.02$ & 19.5 \\
\hline 16 & Mohsin college & $\mathrm{M}_{2}$ & $7.5 \pm 0.02$ & $96 \pm 0.02$ & 21.6 \\
\hline 17 & New Market & $\mathrm{N}_{1}$ & $* 9.1 \pm 0.01$ & $87 \pm 0.02$ & 19.0 \\
\hline 18 & Oxygen & $\mathrm{O}_{1}$ & $7.9 \pm 0.01$ & $152 \pm 0.01$ & 22.5 \\
\hline 19 & Panchlaish & $\mathrm{P}_{1}$ & $* 9.0 \pm 0.02$ & $166 \pm 0.10$ & 18.7 \\
\hline 20 & Rasulbag & $\mathrm{R}_{1}$ & $8.1 \pm 0.10$ & $201 \pm 0.10$ & 19.5 \\
\hline
\end{tabular}

Note: $T D S=$ Total dissolved solids; *= More than recommended by $\mathrm{WHO}$ 
Screening and isolation of bacteria

Table 3 shows that TVC value of drinking water samples within a range of nil to $520 \times 10^{3} \mathrm{cfu} / \mathrm{mL}$. Among $85 \%$ of positive water samples, $65 \%$ of them had higher TVC values (>500 cfu/mL) stated as US EPA standard, while $50 \%$ of total samples could not meet the BDS (>1000 cfu/mL) and WHO standard recommended value $(>1000 \mathrm{cfu} / \mathrm{mL})$ (Sarker et al. 2016, WHO 1996). But, TVC only cannot determine the risk and severity as well as the presence of pathogenic and faecal colifrom origin of bacteria; thus, further microbiological analysis was performed (Table 3), where, $70 \%$ and $50 \%$ of the total samples were found to be contaminated with coliform and faecal

Table 3. Microbiological contamination in drinking water samples

\begin{tabular}{|c|c|c|c|c|c|c|c|}
\hline \multirow[b]{2}{*}{$\begin{array}{c}\text { Sample } \\
\text { ID }\end{array}$} & \multirow{2}{*}{$\begin{array}{c}\text { Total } \\
\text { Viable } \\
\text { Count } \\
\text { (TVC) } \\
\text { cfu/ml }\end{array}$} & \multirow{2}{*}{$\begin{array}{c}\text { Total } \\
\text { Coliform } \\
\text { Count }(\mathrm{TCC}) \\
\text { MPN/100ml }\end{array}$} & \multirow{2}{*}{$\begin{array}{c}\text { Total Faecal } \\
\text { Coliform } \\
\text { Count } \\
\text { (TFCC) } \\
\text { MPN/100ml }\end{array}$} & \multirow{2}{*}{$\begin{array}{c}\text { Total } \\
\text { Staphylococci } \\
\text { Count (TSC) } \\
\text { cfu/ml }\end{array}$} & \multicolumn{3}{|c|}{ Presence of } \\
\hline & & & & & $\begin{array}{l}\text { Salmone } \\
\text { lla spp. }\end{array}$ & $\begin{array}{l}\text { Shigell } \\
a \text { spp. }\end{array}$ & $\begin{array}{c}\text { Vibrio } \\
\text { spp. }\end{array}$ \\
\hline $\mathrm{A}_{1}$ & $4.1 \times 10^{3}$ & $>1100$ & $>1100$ & $0.3 \times 10^{3}$ & + & - & + \\
\hline $\mathrm{A}_{2}$ & $360 \times 10^{3}$ & 240 & 240 & 0 & - & - & + \\
\hline $\mathrm{B}_{1}$ & $200 \times 10^{3}$ & 4 & 4 & 0 & + & - & + \\
\hline $\mathrm{C}_{1}$ & $11.1 \times 10^{3}$ & 4 & 0 & $0.2 \times 10^{3}$ & - & - & + \\
\hline $\mathrm{C}_{2}$ & $0.2 \times 10^{3}$ & 0 & 0 & 0 & - & - & - \\
\hline $\mathrm{D}_{1}$ & 0 & 0 & 0 & 0 & - & - & - \\
\hline $\mathrm{E}_{1}$ & $5 \times 10^{3}$ & 23 & 23 & 0 & - & - & + \\
\hline $\mathrm{G}_{1}$ & $28.2 \times 10^{3}$ & 93 & 93 & 0 & - & - & + \\
\hline $\mathrm{H}_{1}$ & 0 & 0 & 0 & 0 & - & - & - \\
\hline $\mathrm{K}_{1}$ & $0.9 \times 10^{3}$ & 460 & 0 & $1.0 \times 10^{3}$ & + & - & + \\
\hline $\mathrm{K}_{2}$ & $0.3 \times 10^{3}$ & 23 & 0 & 0 & - & - & + \\
\hline $\mathrm{K}_{3}$ & $0.8 \times 10^{3}$ & 23 & 9 & 0 & - & - & + \\
\hline $\mathrm{L}_{1}$ & $0.3 \times 10^{3}$ & 23 & 23 & 0 & - & - & + \\
\hline $\mathrm{L}_{2}$ & $4.5 \times 10^{3}$ & 9 & 9 & 0 & - & - & + \\
\hline $\mathrm{M}_{1}$ & $520 \times 10^{3}$ & 9 & 0 & $0.1 \times 10^{3}$ & + & - & - \\
\hline $\mathrm{M}_{2}$ & $0.4 \times 10^{3}$ & 0 & 0 & 0 & + & - & + \\
\hline $\mathrm{N}_{1}$ & $18 \times 10^{3}$ & 93 & 93 & 0 & - & - & + \\
\hline $\mathrm{O}_{1}$ & $0.7 \times 10^{3}$ & 9 & 9 & 0 & - & - & + \\
\hline $\mathrm{P}_{1}$ & 0 & 0 & 0 & 0 & - & - & - \\
\hline $\mathrm{R}_{1}$ & $70 \times 10^{3}$ & 0 & 0 & 0 & + & - & + \\
\hline $\begin{array}{c}\text { Contamina } \\
\text { tion }(\%)\end{array}$ & $85 \%$ & $70 \%$ & $50 \%$ & $20 \%$ & $30 \%$ & $0 \%$ & $75 \%$ \\
\hline
\end{tabular}


coliform bacteria, respectively; which failed to meet the WHO drinking water standards (WHO 1996, Oyedeji et al. 2010). The prevalence of indicator organisms in water constitutes a serious threat to the community and the processors and handlers asked for good water manufacturing practices (Mina et al. 2018). During TSC count, $20 \%$ of the samples were found contaminated with Staphylococcus aureus proved by the formation of yellow colonies on MSA agar media. Then, among twenty water samples; 6 (30\%) samples were found to be contaminated with Salmonella spp., $15(75 \%)$ samples with Vibrio spp. (Table 3) and no $(0 \%)$ detectable Shigella spp. was found.

\section{Biochemical characterization and identification}

Among twenty (20) samples, forty three bacterial positive isolates were subjected to biochemical tests by growing them on different media (Table 4). 38 isolates were identified as gram negative by detecting peptidoglycan present in a thick layer in bacteria while the rest of 5 isolates were gram positive (Burke and Pister 1986). In our study most of the isolates (28) were oxidase negative, except fifteen (Beveridge 2001). All of the isolates were found catalase positive in this study by producing catalase enzyme (Taylor and Achanzar 1972). During citrate utilization, 38 isolates were citrate positive except five, which are not capable to utilize citrate as a carbon source (Koser1924). During indole test, only 22 isolates were found positive as having the capability to degrade amino acid (tryptophan) and produce indole (Holding and Collee 1971). Positive results were found for 33 isolates during methyl-red test; and six positive results were also found in case of voges-proskauer (VP) test as they have the capability to produce acetylmethyl carbinol from glucose fermentation except most (37) of the isolates were VP negative (Holding and Collee 1971).

Then, visual identification was done for coliform and faecal coliform on EMB agar media (Table 4), where four isolates produced green metallic sheen and thus identified as $E$. coli; six isolates were identified as Klebsiella pneumoniae as they produced large moist, shiny, mucoid pink colony; and two isolates were identified as Citrobacter spp. as they produced flat colonies which are dark violet in colour. Colony morphology was studied on TCBS agar and biochemical properties, where five isolates were identified as Vibrio cholerae and the rest ten were identified as other Vibrio spp. For further confirmation of Vibrio spp. and Salmonella spp. triple sugar iron (TSI) agar test was performed (Table 4) which confirms fifteen isolates as Vibrio cholerae and other Vibrio spp. as they produced acidic slant and butt. And in case of Acinetobacter spp., both of the slant and butt became alkaline, whereas Salmonella spp. produced alkaline slant and acidic butt (APHA 2001). Besides, 4 (20\%) isolates were formed yellow colonies on MSA media which indicates those as Staphylococcus aureus. Micrococcus spp. 1 (5\%) was identified by gram staining and biochemical properties, as it did not grow in any selective and differential media (Table 4) (Bergey et al. 1974).

Depending on biochemical characterization of specific bacterial isolates, total 43 (forty three) bacterial isolates were provisionally identified (Cowan 1974, Bergey et al. 1974) belongs to 9 different species as: Escherichia coli, Citrobacter spp., Klebsiella pneumoniae, Vibrio cholerae, Vibrio spp., Acinetobacter spp., Salmonella spp., Staphylococcus aureus and Micrococcus spp. (Table 4). 
Table 4. Morphological and biochemical characteristics of bacterial isolates

\begin{tabular}{|c|c|c|c|c|c|c|c|c|c|}
\hline Isolate(s) & $\begin{array}{c}\text { Gram } \\
\text { staining }\end{array}$ & Oxidase & Catalase & Citrate & Indole & $\begin{array}{c}\text { MR } \\
\text { (Methyl } \\
\text { red) }\end{array}$ & $\begin{array}{c}\text { VP } \\
\text { (voges- } \\
\text { proskauer) }\end{array}$ & $\begin{array}{c}\text { TSI } \\
\text { (slant/ } \\
\text { butt) }\end{array}$ & $\begin{array}{c}\begin{array}{c}\text { Provisionally } \\
\text { identified } \\
\text { Bacteria }\end{array} \\
\text { (Ref.: Lower etal. } \\
\text { 2001; } \\
\text { Cowan 1974; } \\
\text { Bergey et al. 1974) }\end{array}$ \\
\hline $\mathrm{L}_{1} \mathrm{C}$ & G - & - & + & - & + & + & - & ND & Eschercichia coli \\
\hline $\mathrm{N}_{1} \mathrm{C}$ & G - & - & + & - & + & + & - & ND & Eschercichia coli \\
\hline $\mathrm{E}_{1} \mathrm{C}$ & G - & - & + & - & + & + & - & ND & Eschercichia coli \\
\hline $\mathrm{O}_{1} \mathrm{C}$ & G - & - & + & - & + & + & - & ND & Eschercichia coli \\
\hline $\mathrm{K}_{2} \mathrm{C}$ & G - & - & + & + & + & + & - & ND & Citrobacter spp \\
\hline $\mathrm{M}_{1} \mathrm{C}$ & G - & - & + & + & + & + & - & ND & Citrobacter spp. \\
\hline $\mathrm{L}_{2} \mathrm{C}$ & G - & - & + & + & - & + & - & ND & $\begin{array}{c}\text { Klebsiella } \\
\text { pneumoniae }\end{array}$ \\
\hline $\mathrm{K}_{1} \mathrm{C}$ & G - & - & + & + & - & + & - & ND & $\begin{array}{c}\text { Klebsiella } \\
\text { pneumoniae }\end{array}$ \\
\hline $\mathrm{G}_{1} \mathrm{C}$ & G - & - & + & + & - & + & - & ND & $\begin{array}{c}\text { Klebsiella } \\
\text { pneumoniae }\end{array}$ \\
\hline $\mathrm{K}_{3} \mathrm{C}$ & G - & - & + & + & - & + & - & ND & $\begin{array}{c}\text { Klebsiella } \\
\text { pneumoniae }\end{array}$ \\
\hline $\mathrm{A}_{2} \mathrm{C}$ & G - & - & + & + & - & - & + & ND & $\begin{array}{c}\text { Klebsiella } \\
\text { pneumoniae }\end{array}$ \\
\hline $\mathrm{A}_{1} \mathrm{C}$ & G - & - & + & + & - & + & - & ND & $\begin{array}{c}\text { Klebsiella } \\
\text { pneumoniae }\end{array}$ \\
\hline $\mathrm{L}_{2} \mathrm{~T}$ & G - & + & + & + & + & - & - & $\mathrm{A} / \mathrm{A}$ & Vibrio cholerae \\
\hline $\mathrm{A}_{2} \mathrm{~T}$ & G - & + & + & + & + & + & - & $\mathrm{A} / \mathrm{A}$ & Vibrio cholerae \\
\hline $\mathrm{O}_{1} \mathrm{~T}$ & G - & + & + & + & + & - & + & $\mathrm{A} / \mathrm{A}$ & Vibrio cholerae \\
\hline $\mathrm{E}_{1} \mathrm{~T}$ & G - & + & + & + & + & - & - & $\mathrm{A} / \mathrm{A}$ & Vibrio cholerae \\
\hline $\mathrm{K}_{1} \mathrm{~T}$ & G - & + & + & + & + & - & + & $\mathrm{A} / \mathrm{A}$ & Vibrio cholerae \\
\hline $\mathrm{K}_{2} \mathrm{~T}$ & G - & + & + & + & + & - & - & $\mathrm{A} / \mathrm{A}$ & Vibrio spp. \\
\hline $\mathrm{M}_{2} \mathrm{~T}$ & G - & + & + & + & + & + & - & $\mathrm{A} / \mathrm{A}$ & Vibrio spp. \\
\hline $\mathrm{N}_{1} \mathrm{~T}$ & G - & + & + & + & + & - & - & $\mathrm{A} / \mathrm{A}$ & Vibrio spp. \\
\hline $\mathrm{L}_{1} \mathrm{~T}$ & G - & + & + & + & + & + & - & $\mathrm{A} / \mathrm{A}$ & Vibrio spp. \\
\hline
\end{tabular}

Table 4 (Contd.) 


\begin{tabular}{|c|c|c|c|c|c|c|c|c|c|}
\hline $\mathrm{C}_{1} \mathrm{~T}$ & $\mathrm{G}-$ & + & + & + & + & + & + & $\mathrm{A} / \mathrm{A}$ & Vibrio spp. \\
\hline $\mathrm{R}_{1} \mathrm{~T}$ & G - & + & + & + & + & - & - & $\mathrm{A} / \mathrm{A}$ & Vibrio spp. \\
\hline $\mathrm{B}_{\mathrm{I}} \mathrm{T}$ & $\mathrm{G}-$ & + & + & + & + & + & - & $\mathrm{A} / \mathrm{A}$ & Vibrio spp. \\
\hline $\mathrm{A}_{1} \mathrm{~T}$ & G - & + & + & + & + & + & + & $\mathrm{A} / \mathrm{A}$ & Vibrio spp. \\
\hline $\mathrm{G}_{1} \mathrm{~T}$ & G - & + & + & + & + & + & - & $\mathrm{A} / \mathrm{A}$ & Vibrio spp. \\
\hline $\mathrm{K}_{3} \mathrm{~T}$ & G - & + & + & + & + & + & + & $\mathrm{A} / \mathrm{A}$ & Vibrio spp. \\
\hline $\mathrm{E}_{1} \mathrm{~S}$ & G - & - & + & + & - & + & - & $\mathrm{K} / \mathrm{K}$ & $\begin{array}{c}\text { Acinetobacter } \\
\text { spp. }\end{array}$ \\
\hline $\mathrm{C}_{1} \mathrm{~S}$ & G - & - & + & + & - & - & - & $\mathrm{K} / \mathrm{K}$ & $\begin{array}{c}\text { Acinetobacter } \\
\text { spp. }\end{array}$ \\
\hline $\mathrm{K}_{2} \mathrm{~S}$ & G - & - & + & + & - & + & - & $\mathrm{K} / \mathrm{K}$ & $\begin{array}{c}\text { Acinetobacter } \\
\text { spp. }\end{array}$ \\
\hline $\mathrm{N}_{1} \mathrm{~S}$ & $\mathrm{G}-$ & - & + & + & - & + & - & $\mathrm{K} / \mathrm{K}$ & $\begin{array}{c}\text { Acinetobacter } \\
\text { spp. }\end{array}$ \\
\hline $\mathrm{L}_{2} \mathrm{~S}$ & G - & - & + & + & - & + & - & $\mathrm{K} / \mathrm{K}$ & $\begin{array}{c}\text { Acinetobacter } \\
\text { spp. }\end{array}$ \\
\hline $\mathrm{M}_{1} \mathrm{~S}$ & G - & - & + & + & - & + & - & $\mathrm{K} / \mathrm{A}$ & Salmonella spp. \\
\hline $\mathrm{K}_{1} \mathrm{~S}$ & G - & - & + & + & - & + & - & $\mathrm{K} / \mathrm{A}$ & Salmonella spp. \\
\hline $\mathrm{B}_{1} \mathrm{~S}$ & $\mathrm{G}-$ & - & + & + & - & + & - & $\mathrm{K} / \mathrm{A}$ & Salmonella spp. \\
\hline $\mathrm{R}_{1} \mathrm{~S}$ & G - & - & + & + & - & + & - & $\mathrm{K} / \mathrm{A}$ & Salmonella spp. \\
\hline $\mathrm{A}_{1} \mathrm{~S}$ & $\mathrm{G}-$ & - & + & + & - & + & - & $\mathrm{K} / \mathrm{A}$ & Salmonella spp. \\
\hline $\mathrm{M}_{2} \mathrm{~S}$ & G - & - & + & + & - & - & - & $\mathrm{K} / \mathrm{A}$ & Salmonella spp. \\
\hline $\mathrm{A}_{1} \mathrm{MY}$ & $\mathrm{G}+$ & - & + & + & - & + & - & ND & $\begin{array}{c}\text { Staphylococcus } \\
\text { aureus }\end{array}$ \\
\hline $\mathrm{C}_{1} \mathrm{MY}$ & $\mathrm{G}+$ & - & + & + & - & + & - & ND & $\begin{array}{c}\text { Staphylococcus } \\
\text { aureus }\end{array}$ \\
\hline $\mathrm{M}_{1} \mathrm{MY}$ & $\mathrm{G}+$ & - & + & + & - & + & - & ND & $\begin{array}{c}\text { Staphylococcus } \\
\text { aureus }\end{array}$ \\
\hline $\mathrm{K}_{1} \mathrm{MY}$ & $\mathrm{G}+$ & - & + & + & - & + & - & ND & $\begin{array}{c}\text { Staphylococcus } \\
\text { aureus }\end{array}$ \\
\hline $\mathrm{C}_{2}$ & $\mathrm{G}+$ & - & + & - & + & + & - & $\mathrm{ND}$ & Micrococcus spp. \\
\hline
\end{tabular}

Note: (+) and (-) indicates positive and negative results respectively; ND= not done (as bacterial isolates except Vibrio spp. Acinetobacter spp. and Salmonella spp. were provisionally identified without TSI test); $A=$ Acidic condition; $K=$ Alkaline condition. 
The overall prevalence showed $20 \%, 30 \%$, $25 \%, 30 \%, 20 \%, 25 \%, 50 \%, 10 \%$ and $5 \%$ of Escherichia coli, Klebsiella pneumoniae, Acinetobacter spp. Salmonella spp., Staphylococcus aureus, Vibrio cholerae, Vibrio spp., Citrobacter spp. and Micrococcus spp., (Fig. 1) respectively, prepared based on the data shown in Table 3 and 4, where few samples showed the presence of Vibrio spp. and Salmonella spp., without indicating the presence of coliform or faecal coliform bacteria as some faecal pathogens may be resistant to disinfection process like chlorination than the indicator bacteria (Sadeghi et al. 2007), is consistent with previous report that the presence or absence of potential pathogens in drinking water did not correlate with any of faecal indicator bacterial concentrations in Australia (Ahmed et al.
Cryptosporidium parvum, Campylobacter jejuni, enterotoxigenic Escherichia coli [ETEC], enteropathogenic E. coli [EPEC], Shigella spp. and Vibrio cholerae $\mathrm{O} 1$ or $\mathrm{O} 139$ (Chauhan et al. 2017). Salmonella spp. specifically S. typhi can cause typhoid fever (WHO 2008, Chauhan et al. 2015) are passed in the faeces and urine, thus people become infected after eating food, drinking water and beverages that have been handled by a person who is infected by contaminated drinking water sewage bacteria (Ashbolt 2004). S. aureus is responsible for several serious health problems including food spoilage, chronic infections, abscesses and wound infection (Chauhan et al. 2015); Klebsiella spp. caused pneumonia and urinary tract infection (WHO 2008). Acinetobacter, is a normal inhabitant of water and sewage environment and can cause urinary tract

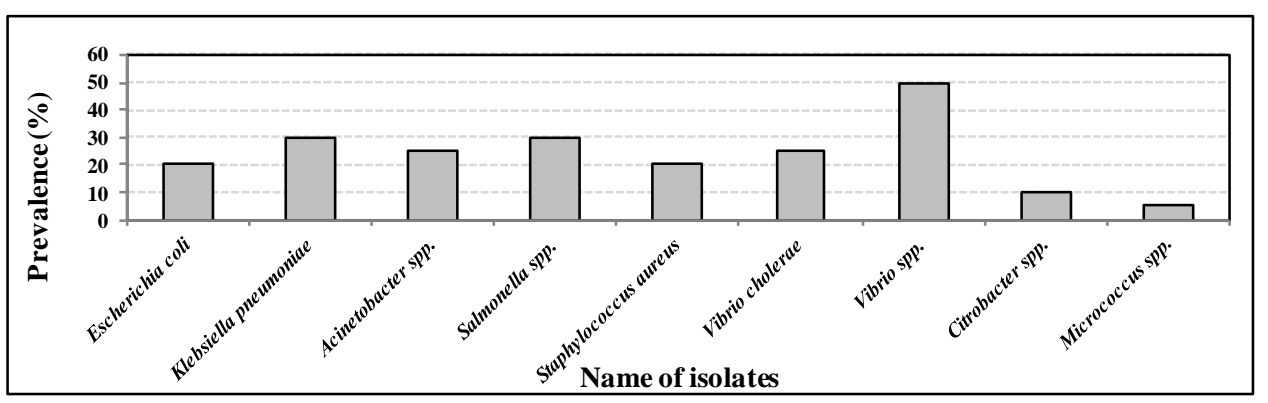

Fig. 1. Prevalence of different human pathogenic bacteria isolated from drinking water samples

2010). It was also found previously, E. coli can disappear in most cases within only four days during experiment of faecal indicator bacteria (Ducluzeau et al. 1976).

The presence of pathogenic bacteria in drinking water is alarming in the perspective of public health as $E$. coli is a leading cause of infantile diarrhea in developing countries. In Bangladesh $75 \%$ of diarrhoeal children and $44 \%$ of control children have an enteric diarrheal pathogen in their stools which are associated with rotavirus, infection, pneumonia, secondary meningitis and wound infection (Chauhan et al. 2015). It is alarming that, most prevalent organism found in this study is Vibrio spp. (75\%) (Fig. 1), which can be responsible for cholera, gastroenteritis, wound infection and bacteraemia (WHO 2008). Moreover, Vibrio cholerae (25\%) can infect the intestine and increase mucous production causing diarrhea with extreme dehydration and, if not treated, death (Howard-Jones 1984). Thus epidemic cholera caused by Vibrio cholerae (O1 and O139) are endemic (Colwell et al. 2003). 
Death occurs in $50-70 \%$ of untreated patients (Faruque et al. 1998) due to devastating watery diarrhea which leads to rapid dehydration which outbreaks a regular seasonal pattern in association with poverty, poor sanitation, unsafe water and inappropriate hygiene in developing regions (Ashbolt 2004).

As unpackaged water is freely available anywhere, presence of pathogenic microorganisms in drinking water can cause various deleterious effects to consumer's health including many diseases which may even cause death leading to a high mortality rate. Diseases like cholera, pneumonia, typhoid etc. are directly linked to contaminated drinking water consumption. Diarrheal diseases weaken the immune system leading to risk of other diseases related to opportunistic infections (Omondi et al. 2015).

\section{Molecular identification of isolated bacteria}

During $16 \mathrm{~S}$ rDNA PCR, all of those nine isolates showed positive bands in agarose gel $(\sim 800$ bp) (Fig. 2a). Homology analysis inferred from 16S rDNA sequence comparison clearly verified that the strains clustered with
Klebsiella pneumonia VM18 $\left(\mathrm{L}_{2} \mathrm{C}\right)$ and Acinetobacter baumannii $\left(\mathrm{K}_{2} \mathrm{~S}\right)$ having $100 \%$ identity in blast analysis (Hasan et al. 2017) and accession number, MG816208 is assigned for Klebsiella pneumoniae VM18 $\left(\mathrm{L}_{2} \mathrm{C}\right)$. Phylogenetic tree was constructed (Fig. 3) to find out similarity with the other member of Klebsiella species with the aid of molecular evolutionary genetic analysis (MEGA 7) by using the neighbor-Joining method (Saitou and Nei 1987), where mostly identical sequences were taken into account.

For the detection of coliforms in drinking water, coliform isolates $(n=12)$ (Table 3 and 4), were then subjected to $l a c Z$ gene amplification (encodes $\beta$-galactosidase protein), where positive bands ( $~ 876 \mathrm{bp})$ in agarose gel were visualized (Fig. 2b). Coliform bacteria are used for monitoring the bacteriological safety of water, on the basis of the realization that the presence of coliform bacteria in water is an indicator of potential human fecal contamination and possible presence of enteric pathogens (Bej et al. 1990, Fatemeh et al. 2014).
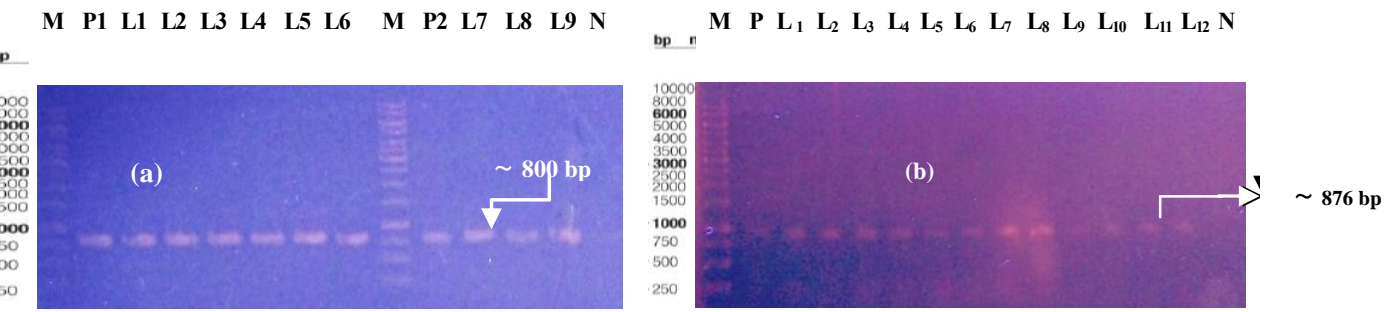

Fig. 2 Electrophoretic (1\% agarose) separation of:

(a) 16S rDNA gene of different isolates. M: marker; P1 and P2: positive control; $\mathrm{N}$ : negative control; L1-L9 ( 800bp): $\mathrm{N}_{1} \mathrm{C}, \mathrm{K}_{2} \mathrm{C}, \mathrm{L}_{2} \mathrm{C}, \mathrm{O}_{1} \mathrm{~T}, \mathrm{C}_{1} \mathrm{~T}, \mathrm{~K}_{2} \mathrm{~S}, \mathrm{~K}_{1} \mathrm{~S}, \mathrm{~K}_{1} \mathrm{MY}$ and $\mathrm{C}_{2}$, respectively.

(b) lacZ gene of different isolates. $\mathrm{M}$ : marker; $\mathrm{P}$ : positive control; $\mathrm{N}$ : negative control; $\mathrm{L}_{1}-\mathrm{L}_{12}$ ( $\sim 876$ bp): $\mathrm{A}_{1} \mathrm{C}, \mathrm{A}_{2} \mathrm{C}, \mathrm{E}_{1} \mathrm{C}, \mathrm{G}_{1} \mathrm{C}, \mathrm{K}_{1} \mathrm{C}, \mathrm{K}_{2} \mathrm{C}, \mathrm{K}{ }_{3} \mathrm{C}, \mathrm{L}_{1} \mathrm{C}, \mathrm{L}_{2} \mathrm{C}, \mathrm{M}_{1} \mathrm{C}, \mathrm{N}_{1} \mathrm{C}$ and $\mathrm{O}_{1} \mathrm{C}$ respectively. 
Table 5. Antibiogram study

\begin{tabular}{|c|c|c|c|c|c|}
\hline Isolate (s) & Bacterial species & $\begin{array}{l}\text { Antibiotic disc } \\
\text { code }\end{array}$ & $\begin{array}{c}\text { Disc } \\
\text { Potency } \\
(\mu \mathrm{g})\end{array}$ & $\begin{array}{c}\text { Zone } \\
\text { ranges } \\
(\mathbf{m m})\end{array}$ & $\begin{array}{c}\begin{array}{c}\text { Interpretation } \\
\text { (S/R/I) }\end{array} \\
\text { (Ref.: Bauer } \text { et al. 1966) }\end{array}$ \\
\hline \multirow[t]{5}{*}{$\mathrm{N}_{1} \mathrm{C}$} & Escherichia coli & AML & 10 & 10 & $\mathrm{R}$ \\
\hline & & CIP & 5 & 25 & $\mathrm{~S}$ \\
\hline & & $\mathrm{CN}$ & 10 & 19 & $\mathrm{~S}$ \\
\hline & & IMP & 30 & 24 & $\mathrm{~S}$ \\
\hline & & LEV & 5 & 23 & $\mathrm{~S}$ \\
\hline \multirow[t]{5}{*}{$\mathrm{K}_{2} \mathrm{C}$} & Citrobacter spp. & AML & 10 & 9 & $\mathrm{R}$ \\
\hline & & CIP & 5 & 25 & $S$ \\
\hline & & $\mathrm{CN}$ & 10 & 17 & $S$ \\
\hline & & IMP & 30 & 24 & S \\
\hline & & LEV & 5 & 23 & $\mathrm{~S}$ \\
\hline \multirow[t]{5}{*}{$\mathrm{L}_{2} \mathrm{C}$} & Klebsiella pneumoniae & AML & 10 & 11 & $\mathrm{R}$ \\
\hline & & CIP & 5 & 27 & S \\
\hline & & $\mathrm{CN}$ & 10 & 21 & S \\
\hline & & IMP & 30 & 29 & S \\
\hline & & LEV & 5 & 28 & $\mathrm{~S}$ \\
\hline \multirow[t]{5}{*}{$\mathrm{O}_{1} \mathrm{~T}$} & Vibrio cholerae & AML & 10 & 7 & $\mathrm{R}$ \\
\hline & & CIP & 5 & 24 & $\mathrm{~S}$ \\
\hline & & $\mathrm{CN}$ & 10 & 18 & $\mathrm{~S}$ \\
\hline & & IMP & 30 & 23 & $\mathrm{~S}$ \\
\hline & & LEV & 5 & 24 & $\mathrm{~S}$ \\
\hline \multirow[t]{5}{*}{$\mathrm{C}_{1} \mathrm{~T}$} & Vibrio. spp. & AML & 10 & 18 & I \\
\hline & & CIP & 5 & 23 & $\mathrm{~S}$ \\
\hline & & $\mathrm{CN}$ & 10 & 19 & $\mathrm{~S}$ \\
\hline & & IMP & 30 & 28 & $S$ \\
\hline & & LEV & 5 & 23 & $\mathrm{~S}$ \\
\hline \multirow[t]{5}{*}{$\mathrm{K}_{2} \mathrm{~S}$} & Acinetobacter spp. & AML & 10 & 16 & I \\
\hline & & CIP & 5 & 25 & $S$ \\
\hline & & $\mathrm{CN}$ & 10 & 18 & S \\
\hline & & IMP & 30 & 27 & S \\
\hline & & LEV & 5 & 26 & S \\
\hline \multirow[t]{5}{*}{$\mathrm{K}_{1} \mathrm{~S}$} & Salmonella spp. & AML & 10 & 19 & S \\
\hline & & CIP & 5 & 24 & S \\
\hline & & $\mathrm{CN}$ & 10 & 21 & $S$ \\
\hline & & IMP & 30 & 28 & S \\
\hline & & LEV & 5 & 23 & $\mathrm{~S}$ \\
\hline \multirow[t]{5}{*}{$\mathrm{K}_{1} \mathrm{MY}$} & Staphylococcus aureus & AML & 10 & 38 & $\mathrm{~S}$ \\
\hline & & CIP & 5 & 37 & S \\
\hline & & $\mathrm{CN}$ & 10 & 31 & S \\
\hline & & IMP & 30 & 49 & S \\
\hline & & LEV & 5 & 38 & S \\
\hline \multirow[t]{5}{*}{$\mathrm{C}_{2}$} & Micrococcus spp. & AML & 10 & 32 & S \\
\hline & & CIP & 5 & 36 & $\mathrm{~S}$ \\
\hline & & $\mathrm{CN}$ & 10 & 30 & S \\
\hline & & IMP & 30 & 45 & $\mathrm{~S}$ \\
\hline & & LEV & 5 & 29 & $\mathrm{~S}$ \\
\hline
\end{tabular}

Note: $\boldsymbol{R}=$ Resistance, $\boldsymbol{S}=$ Susceptible, $\boldsymbol{I}=$ intermediate sensitive, $\boldsymbol{A M L}=$ Amoxicillin,

$\boldsymbol{C I P}=$ Ciprofloxacin, $\boldsymbol{C N}=$ Gentamicin, $\boldsymbol{I M P}=$ Imipenam and $\boldsymbol{L E} \boldsymbol{V}=$ Levofloxacin . 
Antibiogram study

The dissemination of resistance can be very frequent phenomenon if the resistant bacteria are present in water. The emergence of multidrug resistant (MDR) and ESBL (extended spectrum beta lactamases) producing $E$. coli has been reported in numerous studies and become a threat to infectious disease management in Bangladesh (Parvez et al. 2016). So, antibiogram studies of nine selected species were performed using five widely prescribed antibiotics named Amoxicillin (AML), Ciprofloxacin (CIP), Gentamicin (CN), Imipenam (IMP) and Levofloxacin (LEV); where E. coli, Citrobacter spp., K. pneumoniae and Vibrio cholerae were highly resistant to
Amoxicillin; besides, Vibrio spp., Acinetobacter spp. showed intermediate sensitivity and remaining three isolates were highly sensitive to Amoxicillin (Table 5) is consistent with past studies (Parvez et al. 2016). Widely uses of Amoxicillin, not only for treatment purpose but also in veterinary and poultry industries as food additives or others (Parvez et al. 2016) may increase their resistance capacity. A great deal of health threat may arise through the spreading of antibiotic resistance to normal flora or creating favorable environment for opportunistic pathogens. All the bacterial genera exhibited sensitivity to Ciprofloxacin, Gentamicin, Imipenam and Levofloxacin (Table 5) found also previously by Srinu et al. (2012).

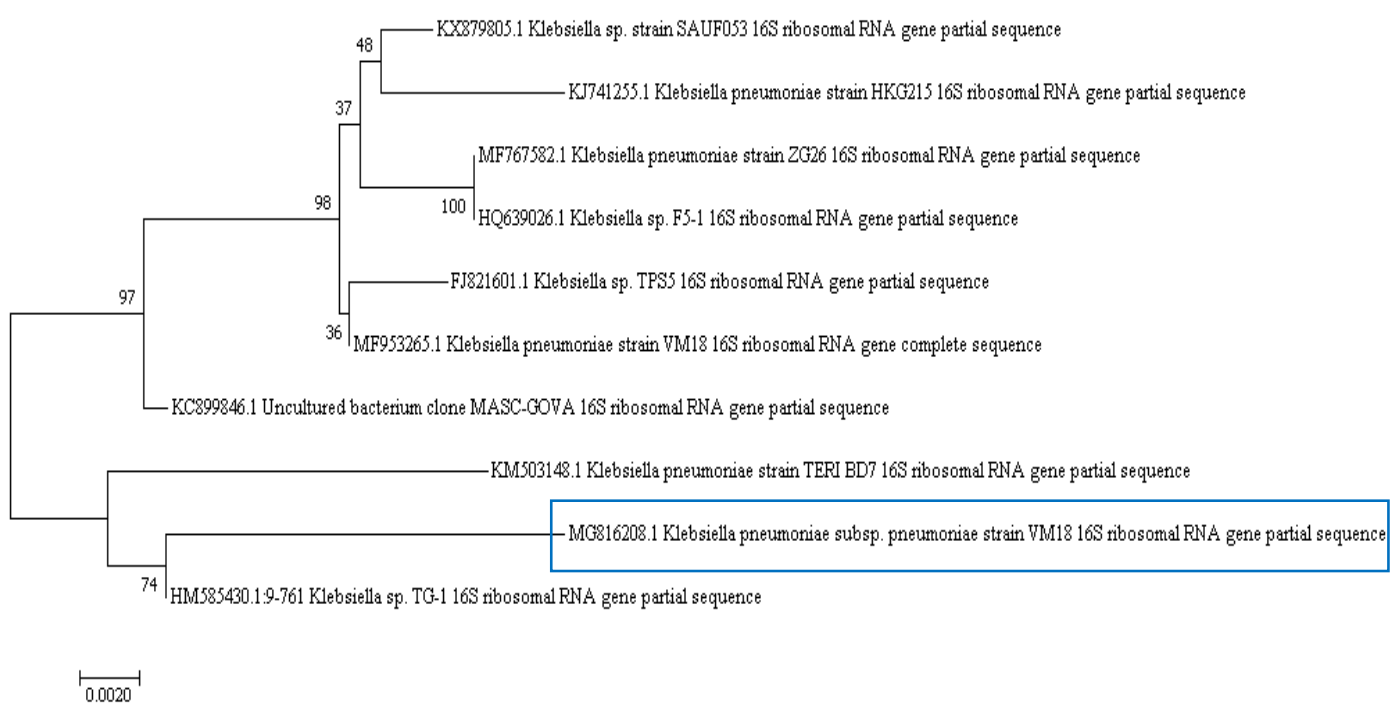

Fig. 3 Evolutionary relationships of taxa: The evolutionary history was inferred using the Neighbor-Joining method (Saitou and Nei 1987). The optimal tree with the sum of branch length = 0.05933807 is shown. The percentage of replicate trees in which the associated taxa clustered together in the bootstrap test (500 replicates) are shown next to the branches (Felsenstein 1985). The tree is drawn to scale, with branch lengths in the same units as those of the evolutionary distances used to infer the phylogenetic tree. The evolutionary distances were computed using the p-distance method (Nei and Kumar 2000) and are in the units of the number of base differences per site. The analysis involved 10 nucleotide sequences. Codon positions included were 1 st $+2 \mathrm{nd}+3 \mathrm{rd}+$ Noncoding. All ambiguous positions were removed for each sequence pair. There were a total of 1473 positions in the final dataset. Evolutionary analyses were conducted in MEGA7 (Kumar et al. 2016). 


\section{CONCLUSION}

This study indicates that unpackaged drinking water available at roadside shops, food vendors, tea stalls in Chittagong city are not safe from microbiological viewpoint (WHO 2008). Thus people of this region are under severe threat of water related diseases and health risk. Though most of the sample's physicochemical properties are within safe zone to drink, but microbiological contamination level has made them impotable. Samples in this study were contaminated with coliform, faecal coliform and pathogenic bacteria like Staphylococcus, Salmonella and Vibrio were supported by both biochemical and molecular methods (Bergey et al. 1974, Fatemeh et al. 2014). The results of antibiogram study found them resistant against some commonly used antibiotics. To detect their contamination rate, the source of water as well as their distribution channels should be monitored and treated on a regular basis. Moreover, it is urgently needed to educate all vendors and shop owners about possible source of contamination and drinking water handling systems. High level of coliform bacteria indicating poor quality of water and presence of antibiotic resistance bacteria is also alarming and need further exploration regarding the source of resistant bacteria. Responsible genes for resistance as proliferation of antibiotic resistant bacteria may hamper the efficacy of antibiotics in chemotherapy (Armstrong et al. 1981). To control adulteration of unpackaged drinking water, social motivation of the consumers and strong supervision by legal authority can play a major role.

\section{ACKNOWLEDGEMENT}

This research was carried out in the Molecular Biology laboratory of the department of Genetic Engineering and Biotechnology, University of
Chittagong and partially funded by the Planning and Development Office of the University of Chittagong, memo no.: 409/P\&D/7$36(19) / 2^{\text {nd }} / 2018$.

\section{Abbreviations}

TAE: Tris-Acetate-EDTA; BLAST: Basic Local Alignment Search Tool; NCBI: National center For biotechnology information; BSTI:

Bangladesh standard and testing institute; EPA:

Environmental protection agency; APHA:

American public health association.

\section{REFERENCES}

Addisu, D. and L. Kiros. 2016. Isolation and identification of bacteria from fresh fruit juices prepared in cafeterias and restaurants, Axum Town, Int. J. Int. Sci. Inn. and Tech. 5(2): 5-10.

Ahmed, W., A. Goonetilleke and T. Gardner. 2010. Implication of faecal indicator bacteria for the microbiological assessment of roofharvested rain water quality in Southeast Queensland, Australia. Can. J. Microbiol. 56: 471-479.

Akuffu I., S. J. Cobbina, E. H. Alhassan and M. Nkoom. 2013. Assessment of the quality of water before and after storage in the Nyankpala community of the TolonKumbungu District, Ghana. Int. J. Sci Technol Res. 2(2): 2277-8616.

American Public Health Association, Compendium of methods for the microbiological examination of foods, APHA, Washington, DC, USA. $4^{\text {th }}$ Ed. 2001.

Armstrong, J. L., D. S. Shigeno, J. Calomiris, R. J. Seidler. 1981. Antibiotic resistant bacteria in drinking water. Appl. Environ. Microbiol. 42: $277-283$. 
Ashbolt N. J. 2004. Microbial contamination of drinking water and disease outcome in developing regions. Toxicology. 198(1-3): 229-238.

Aziz, K. M. A., B. A. Hoque, S. K. M. H. Minnatullah, Z. Hasan, M.K. Patwary, M. M. Rahman, S. Cairncross. 1990. Water supply, sanitation and hygiene education report of health impact study in Mirzapur, Bangladesh.

Bauer, A.W., W.M. Kirby, J. C. Sherris and M. Turck. 1966. Antibiotic susceptibility testing by a standardized single disk method. Ame. J. Clin. Pathol. 45:493-496.

Beiranvand, M., M. Amin, A. HashemiShaharaki, B. Romani, S. Yaghoubi and P. Sadhegi. 2017. Antimicrobial activity of endophytic bacterial populations isolated from medicinal plants of Iran. Iranian $J$ of Microbiol. 9(1): 11-18.

Bej, A. K., R. J. Steffan, J. Di Cesare, L. Haff and R. M. Atlas. 1990. Detection of coliform bacteria in water by polymerase chain reaction and gene probes. Appl. Environ. Microbiol. 56(2): 307-314.

Bergey, D. H., R. E. Buchanan and N. E. Gibbons. 1974. Bergey's manual of determinative bacteriology. Williams and Wilkins Co., Baltimore. 1246.

Beveridge, T. J. 2001. Use of the gram stain in microbiology. Biotech. Histochem. 76(3): 111118.

Burke, B. E. and R. M. Pister. 1986. Cadmium transport by a $\mathrm{Cd}^{2+}$ sensitive and a $\mathrm{Cd}^{2+}$ resistant strain of Bacillus subtilis, Can J Microbial. 32: 539-542.

Cappuccino, J. G. and N. Sherman. 2004. Microbiology a laboratory manual. Pearson Education (Singapore).

Chalchisa, D., M. Megersa and A. Beyene. 2017. Assessment of the quality of the drinking water in storage tanks and its implication on the safety of urban water supply in developing countries. Environ Syst Res. 6(12): 1-6.
Chauhan, A., P. Goyal, A. Varma and T. Jindal. 2017. Microbiological evaluation of drinking water sold by roadside vendors of Delhi, India. Appl. Water Sci. 7(4):16351644.

Collee, J. G., A. G. Frasher, B. P. Marmion and A. Simmons. 1996. Mackie and McCartney Practical Medical Microbiology. Fourteenth edition, Churchill Living Stone.

Colwell, R. R., A. Huq, S. M. Islam, K. M. A. Aziz, M. Yunus, H. N. Khan, A. Mahmud, R.B. Sack, G.B. Nair, J. Chakraborty, D.A. Sack, E. Russek-Cohen. 2003. Reduction of cholera in Bangladeshi villages by simple filtration. Proc. Nat. Acad. Sci. 100(3): 1051-1055.

Cowan, S.T. 1974. Cowan and Steel's manual for the identification of medical bacteria. 2nd Edition, Cambridge University Press, Cambridge, 67-83.

Ducluzeau, R., J. M. Bochand and S. Dufrense. 1976. Longevity of various bacterial strains of intestinal origin in gas free mineral water. Eur. J. Appl. Microbiol. 3: 227-236.

Faruque, S.M., M.J. Albert and J.J. Mekalanos. 1998. Epidemiology, genetics, and ecology of toxigenic Vibrio cholerae (Review). Microbiol Mol Biol Rev. 62(4): 1301.

Fatemeh D., Z. M. Reza, A. Mohammad, K. Salomeah, A. G. Reza and S. Hossein S. 2014. Rapid detection of coliforms in drinking water of Arak city using multiplex PCR method in comparison with the standard method of culture (most probably number). Asian Pac. J. Trop. Biomed. 4(5): 404-409.

Felsenstein J. 1985. Confidence limits on phylogenies: An approach using the bootstrap. Evolution 39:783-791.

Hasan, M. M., L. W. Marzan, A. Hosna, A. Hakim and A. K. Azad. 2017. Optimization of some fermentation conditions for the production of extracellular amylases by using Chryseobacterium and Bacillus isolates from organic kitchen wastes. J. of Gen. Eng. and Biotechnol. 15: 59-68. 
Holding, A. J. and J. G. Collee. 1971. Routine biochemical test, in method in microbiology, (Norris JR and Ribbons DW, eds), Academic Press Inc. Ltd, London, 1-32.

Howard-Jones. N. 1984. Robert Koch and the cholera vibrio: a centenary. Br. Med. J. 288(6414): 379-381.

Koser, S. A. 1924. Differential tests for colonAerogenes group in relation to sanitary quality of water. J. Inf. Dis. 35: 14-22.

Kumar, S. Stecher, G. and Tamura, K. 2016. MEGA7: Molecular Evolutionary Genetics Analysis Version 7.0 for bigger datasets. Molecular Biology and Evolution 33:18701874.

Malik, A. and M. Ahmad. 1994. Incidence of drug and metal resistance in E. coli strains from sewage water and soil, Chem. Environ. Res. 3: 3-11.

Manie, T., V. S. Brozel and W. J. P.A Veith. 1999. Antimicrobial resistance of bacterial flora associated with bovine products in South Africa. J Food Prot, USA. 62(6): 615 618.

Marzan, L. W., M. Hossain, S. A. Mina, Y. Akter and A. M. MA. Chowdhury. 2017. Isolation and biochemical characterization of heavy-metal resistant bacteria from tannery effluent in Chittagong city, Bangladesh: Bioremediation viewpoint. Egyp. J. Agri. Res. 43(1): 65-74.

Mina, S. A., L. W. Marzan, T. Sultana and Y. Akter. 2018. Quality assessment of commercially supplied drinking jar water in Chittagong City, Bangladesh. Appl. water sci. 8(24):1-8.

Muyima, N. Y. O. and F. E. Ngcakani. 1998. Indicator bacteria and re-growth potential of the drinking water in Alice, Eastern Cape. Water SA-Pretoria. 24(1): 29-34.
Nawas, T., R. M. Mazumder, S. Das, M. N. Nipa, S. Islam, H. R. Bhuiyan and I. Ahmed. 2012. Microbiological quality and antibiogram of E. coli, Salmonella and Vibrio of salad and water from restaurants of Chittagong. J. of Environ. Sci. and natural resources. 1: 159-166.

Nei, M. and Kumar, S. 2000. Molecular Evolution and Phylogenetics. Oxford University Press, New York.

Olaoye, O.A. and A.A. Onilude. 2009. Assessment of microbiological quality of sachet-packaged drinking water in Western Nigeria and its public health significance. Public health. 123: 729-734.

Oluwole, O. A., O. M. David, C. R. Falegan, B. Awojuyigbe and O. M. Olajide. 2016 Microbiological and physicochemical properties of commercial seal tampered refrigerated fruit juices. Int $J$ of Biological Res. 4(1): 21-24.

Omondi, O. L., C. Wang, X. Xue and O. G. Lawi. 2015. Modeling of the effects of vaccination on rotavirus infection. $A d v$. in Diff. Equations. 381: 1-12.

Oyedeji, O., P. O. Olutiola and M. A. Moninuola. 2010. Microbiological quality of packaged drinking water brands marketed in Ibadan metropolis and Ile-lfe city in South Western Nigeria. Afr. J. of Micro. Res. 4(1): 96-102.

Parvez, A. K., S. M. Liza, M. Marzan, A. Ahmed and M.S. Rahman. 2016. Bacteriological quality of drinking water samples across Bangladesh. Arch of clinical micro. 1:9.

Prasai, T., B. Lekhak, D. R. Joshi and M. P. Baral. 2007. Microbiological analysis of drinking water of Kathmundu valley. Scientific world. 5(5): 112-114. 
Rashed, M., M., U., Aftab, M. H. Azizul, K. M. Saurab, A. Mrityunjoy, M. R. Majibur. 2013. A microbiological study of vendor and packed fruit juices locally available in Dhaka city, Bangladesh. Int Food Res J. 20(2): 1011-1015.

Sadeghi, G. H., M. Mohammadian, M., Nourani, M. Peyeda and A. Eslami. 2007. Microbiological quality assessment of rural drinking water supplies in Iran. J of Agri. and Social Sci. 3: 31-33.

Saitou, N. and Nei, M 1987. The neighborjoining method: A new method for reconstructing phylogenetic trees. Mol. Biol. and Evol. 4: 406-425.

Sambrook, J., E. F. Fritsch and T. Maniatis. 1989. Molecular Cloning: A Laboratory Manual. $2^{\text {nd }}$ Ed. New York: Cold Spring Harbor Laboratory Press. 1989.

Sarker, A., S. Dash, M. M. Hoque, S. Ahmed and R. Shaheb. 2016. Assessment of microbial quality of water in popular restaurants in Sylhet city of Bangladesh. Bang. J. Agr. Res. 41(1): 115-125.

Srinu, B., A. Vijaya Kumar, E. Kumar and T. Madhava Rao. 2012. Antimicrobial resistance of bacterial foodborne pathogens, J Chem. and Pharm. Res. 4(7): 3734- 3736.

Tasnim, F., H. M. Anwar, S. Nusrath, H. M. Kamal, D. Lopa and H. K. M. Formuzul 2010. Quality assessment of industrially processed fruit juices available in Dhaka city, Bangladesh. Mal. J. Nutr 16(3): 431438.

Taylor, W. I. and D. Achanzar. 1972. Catalase test as an aid to the identification of enterobacteriaceae, J. Appl. Microbiol. 24: 58-61.
Uddin, M. A., M. Akrong, M. H. Asmah, R. A. Banu and E. D. O. Ansa. 2011. Isolation and identification of pathogenic Escherichia coli, Klebsiella spp. and Staphylococcus spp. in raw milk samples collected from different areas of Dhaka city, Bang. Stam. J. microbial. 1(1): 19-23.

UNCED. 1992. Protection of the quality and supply of freshwater resources: application of integrated approaches to the development, management and use of water resources. The United Nations conference on environment and development. Chapter 18, agenda 21

WHO. 1996. Guidelines for drinking water quality $2^{\text {nd }}$ edition. Volume II, Health criteria and other supporting information. World Health Organization, Geneva.

WHO. 2008. Guidelines for drinking water quality, incorporating $1^{\text {st }}$ and $2^{\text {nd }}$ Addenda, volume 1, recommendations, $3^{\text {rd }}$ ed. World Health Organization; Geneva, Switzerland.

WHO. 2015. Drinking water fact sheet $\mathrm{N}^{\circ} 391$. http://www.who.int/mediacentre/factsheets/fs391/en/. World Health Organization. Accessed Feb 2016.

Williams, S. T. 1989. Bergey's manual of systematic bacteriology. Williams and Wilkins, Baltimore.

Zuthi, M. F. R., M. Biswas and M. N. Bahar. 2009. Assessment of supply water quality in the Chittagong city of Bangladesh. ARPN J Eng App Sci. 4:73-80.

(Received revised manuscript on 24 October 2018) 